\title{
Un regard sur l'avenir des neurosciences cliniques
}

\author{
Anton Valavanis ${ }^{a}$, Claudio Bassetti ${ }^{b}$, Karl Schaller \\ a Prof. Dr méd., Zurich, Past-Président SFCNS, membre FMH \\ ${ }^{b}$ Prof. Dr méd., Berne, Président SFCNS de 2009 à 2013, membre FMH \\ c Prof. Dr méd., Genève, Président SFCNS, membre FMH
}

Lorsque la Swiss Federation of Clinical Neuro-Societies (SFCNS) a été créée il y a 7 ans, l'un des principaux objectifs était non seulement de combattre la fragmentation croissante au sein des neurosciences cliniques, mais également de renforcer l'interaction entre les nombreuses branches de la neuroscience clinique moderne. L'idée était de donner aux neurocliniciens la possibilité d'échanger davantage sur les sujets d'intérêt communs sans pour autant faire obstacle à la spécialisation nécessaire. La SFCNS a donc l'échange interdisciplinaire dans le sang. Rien que pour cette raison, la fédération considère de sa responsabilité d'organiser régulièrement un congrès offrant l'opportunité d'une telle rencontre. En 2016 s'est donc tenu, du 28 au 30 septembre, le $3^{\mathrm{e}}$ Congrès SFCNS, à Bâle, où avait déjà eu lieu la première édition.

\section{Spectre thématique agrandi}

Environ 670 intéressés et professionnels du domaine des neurosciences cliniques ont participé fin septembre à ce congrès triennal. Depuis la première édition du congrès en 2010 , le spectre thématique s'est continuellement élargi. Pour le président de la SFCNS,

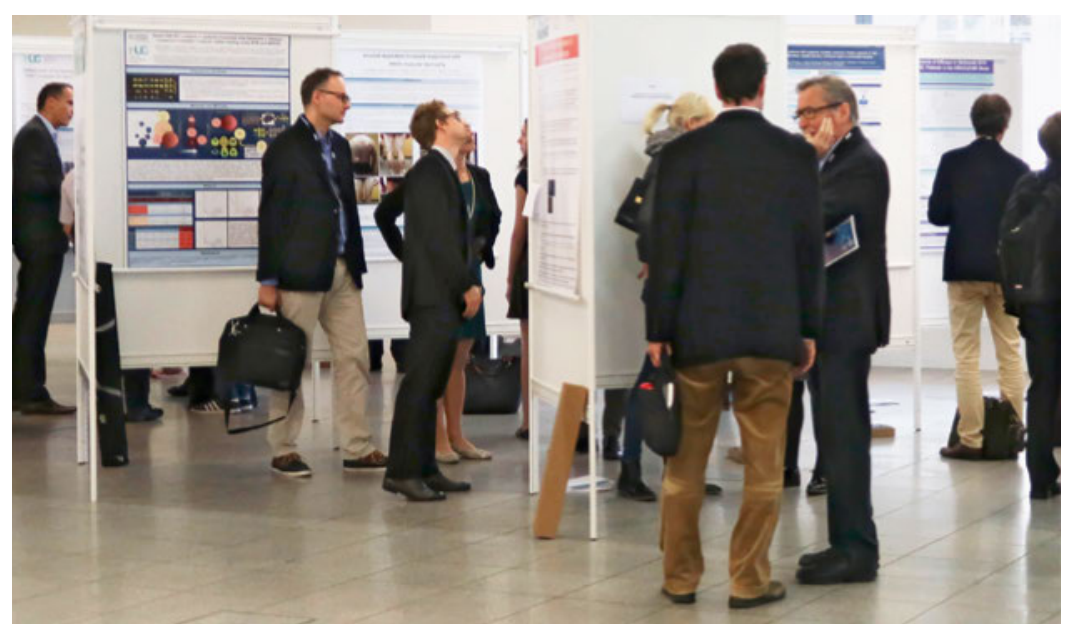

Quelle direction pourraient prendre à l'avenir les neurosciences cliniques suisses sur le plan professionnel, ainsi que sur le plan de la politique de santé? Au congrès de septembre 2016, les participants ont discuté cette question de manière intensive.
Anton Valavanis, de Zurich, le programme de cette année, élaboré par le comité scientifique sous la direction de Claudio Bassetti, de Berne, reflétait pleinement la diversité de la SFCNS, composée d'un total de 14 sociétés de neurosciences. Outre des thèmes principaux médicaux, tels que la neurogénétique, le sommeil et l'épilepsie, la neuro-immunologie, la neuro-oncologie, les accidents vasculaires cérébraux et la douleur, le congrès de cette année avait également pour but, dans le cadre de six manifestations se déroulant pour certaines en parallèle, de discuter de la direction que pourraient ou devraient prendre à l'avenir les neurosciences cliniques suisses sur le plan professionnel, ainsi que sur le plan de la politique de santé et de la politique professionnelle.

Au cours de son dernier discours d'ouverture, Anton Valavanis, remplacé à son poste de président de la société par Karl Schaller, de Genève, lors de l’assemblée générale de la SFCNS, a fourni un aperçu de cet avenir en présentant les projets actuels et futurs de la fédération.

\section{Common Trunk: entrer en contact avec les sous-disciplines dès le début de la formation postgraduée}

L'un des grands projets de la SFCNS, qui s'inscrit tout à fait dans la tradition interdisciplinaire de la fédération en tant qu'intermédiaire, est l'introduction d'un tronc commun, ou common trunk, dans la formation postgraduée en neurosciences cliniques. Les jeunes neuroscientifiques de Suisse doivent ainsi entrer en contact avec les nombreuses sous-disciplines des neurosciences cliniques, et ce dès le début de leur formation postgraduée. De la neurologie à la neuropédiatrie en passant par la neuroradiologie et la neurochirurgie, toutes les neurodisciplines cliniques doivent être représentées dans ce tronc commun sous une forme adaptée. L'objectif est de former les meilleurs professionnels. «Il est clair que seules les futures générations de neurocliniciens profiteront de cette offre, mais il est de notre devoir de poser les bases d'un tel projet», souligne Anton Valavanis. 


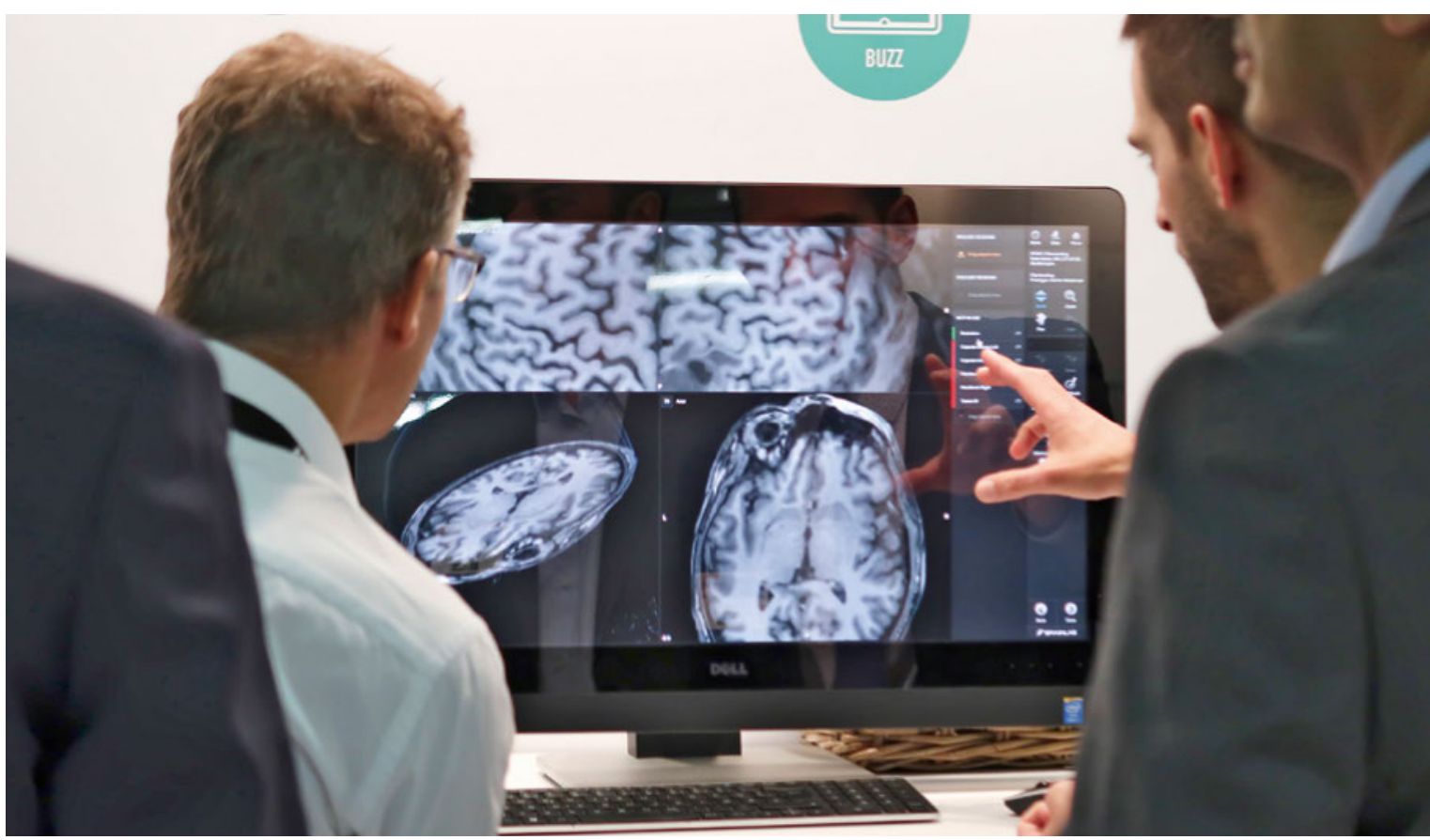

Les neuroscientifiques suisses doivent entrer en contact avec les sous-disciplines des neurosciences cliniques, dont par exemple la neuroradiologie, dès le début de leur formation postgraduée.

Une autre étape essentielle pour une promotion efficace de la relève est la création d'une Federation for Young Clinical Neuroscientists, dans laquelle la jeune génération de neurocliniciens peut échanger. Le président en exercice de la Swiss Association of Young Neurologists (SAYN), Arseny Sokolov, de Lausanne, prend la présidence de ce réseau. La Summer School de la SFCNS, qui se tiendra pour la toute première fois en août prochain à l'Université de Zurich, devra encore davantage favoriser l'échange. Elle abordera principalement des sujets tels que la neuro-immunologie, les maladies neurovasculaires, la neurodégénérescence et la neuro-oncologie.

Un autre projet qui sera prochainement lancé par la SFCNS est la création du journal en ligne Clinical and Translational Neuroscience, soutenu par de nombreux neurocentres suisses, par des neurosociétés, ainsi que par certaines fondations et des partenaires de l'industrie. Ce nouveau journal gratuit, qui devrait paraître à hauteur de quatre à six numéros par an, s'adresse à tous les acteurs des neurosciences cliniques.

\section{Plus de reconnaissance en matière de politique de santé pour la neuroradiologie}

Avec les années, les neurosciences cliniques, qui étaient jusqu'à présent plutôt contemplatives, sont devenues une discipline dans laquelle les urgences, telles que les hémorragies intracrâniennes et les accidents vasculaires cérébraux, déterminent souvent le quotidien dans une clinique. Dès 2011, la SFCNS s'est vue confier le mandat de coordonner les processus de certification des centres de compétence hautement spécialisés dans les accidents vasculaires cérébraux (les Stroke centers et les Stroke units). Un groupe de travail de la SFCNS spécialement créé à cet effet se consacrera désormais exclusivement à la neuromédecine intensive. Tout à fait dans l'esprit de la SFCNS, ce groupe de travail sera lui aussi fondamentalement axé sur l'interdisciplinarité.

\section{La SFCNS a l'échange interdisciplinaire dans le sang.}

Aider la neuroradiologie - l'une des disciplines clés des neurosciences cliniques et l'une des disciplines fondatrices de la fédération - à gagner plus de reconnaissance en matière de politique de santé est une priorité majeure de longue date de la SFCNS. Le fait que la neuroradiologie en tant que discipline ne dispose aujourd'hui d'encore aucun titre de spécialiste, malgré son influence croissante et sa grande importance indiscutable, n'est pas adapté à l'époque et est par conséquent insatisfaisant. Dans les prochains temps, la SFCNS souhaite s'engager encore plus afin que bientôt, les premiers jeunes neuroradiologues puissent passer un diplôme de médecin spécialiste dans cette neurodiscipline établie et attractive pour la relève.

\section{Crédits photo}

SFCNS 